Research paper

\title{
Effect of clay mineral addition on properties of bio-based polymer blends
}

\author{
Ana S. Abreu *, M. Oliveira, A.V. Machado \\ Institute of Polymers and Composites (IPC), Institute of Nanostructures Nanomodelling and Nanofabrication (I3N), University of Minho, Campus de Azurém, 4800-058 Guimarães, Portugal
}

\section{A R T I C L E I N F O}

\section{Article history:}

Received 24 May 2014

Received in revised form 2 December 2014

Accepted 5 December 2014

Available online 18 December 2014

\section{Keywords:}

Organoclay

Sustainability

Thermoplastic starch

Packaging applications

Biodegradability

\begin{abstract}
A B S T R A C T
The effect of clay mineral addition to bio-based blends on morphology and physical properties of thermoplastic starch (TPS) and polypropylene grafted with maleic anhydride (PP-g-MA) was investigated. Blends and nanocomposites containing organoclay, Cloisite 30B, were prepared by melt mixing and characterized by several techniques. X-ray diffraction (XRD), scanning and transmission electron microscopy (SEM, STEM) and dynamic mechanical analysis (DMA) demonstrate a very good dispersion of the clay mineral in the polymer matrix, an increase of polymer compatibility and an improvement of mechanical properties. Biodegradation studies performed in compost revealed that Cloisite 30B addition enhanced the matrix biodegradability.

Therefore clay minerals, which can be obtained from natural resources, can be efficiently used to improve the properties of bio-based materials and contribute to sustainability.
\end{abstract}

(c) 2014 Elsevier B.V. All rights reserved.

\section{Introduction}

The consumption increase of synthetic plastic had led to serious ecological problems due to their non-biodegradability. Although their complete replacement by eco-friendly polymers is not yet possible to achieve, at least for specific applications the use of biodegradable polymers became crucial. Therefore, nowadays there is a great motivation to develop sustainable materials and innovative process technologies that can reduce the dependence on fossil fuel and the amount of residue after lifetime. Bio-based nanocomposites are expected to possess improved strength and stiffness with little sacrifice of toughness, reduced gas/water vapor permeability, lower coefficient of thermal expansion, and an increased heat deflection temperature. These characteristics open an opportunity for the use of new, high performance, lightweight green nanocomposite to replace conventional petroleum-based materials (Tang and Alavi, 2011). Thus, the development of more environmentally friendly polymeric materials from bio-based sources with improved physical and mechanical properties, at very low filler loadings, has become the focus of significant research attention (Ojijo and Sinha Ray, 2013; Rhim et al., 2013).

Preparation of bio-based polymer nanocomposites involves the incorporation of clays within the polymer matrix to produce a material that has a nano-level particle dispersion, which leads to a considerable change of the matrix properties. Among various promising types of nanoscale fillers are layered silicate clay minerals, such as, montmorillonite and kaolinite (Rhim et al., 2013). To produce nanocomposites with desired properties, a good dispersion is required. Therefore the clay minerals are organically modified to induce affinity with the

\footnotetext{
* Corresponding author. Tel.: + 351253 510320; fax: + 351253510339.

E-mail address: anasofiaabreu@dep.uminho.pt (A.S. Abreu).
}

polymer matrix (Kráčalík et al., 2007). During nanocomposites preparation by melt compounding initially an intercalation process of the polymer macromolecules into the interlayer spaces occurs and finally clay mineral exfoliation in the polymer matrix. This process is diffused control and requires a balance between shear rate and residence time to reach complete exfoliation. However, these requirements have some drawbacks, since polymer degradation can also take place.

Thermoplastic starch (TPS) is one of the most promising biodegradable polymer, it is completely degradable in soil and water and when blended with a non-biodegradable polymer it promotes the biodegradability of the latter. TPS is obtained through plasticization of starch and typically blended with another hydrophobic polymer due to its hygroscopic nature (Hottle et al., 2013; Mohammadi Nafchi et al., 2013). Since, most of times, TPS cannot meet all the requirements of a packaging material, it is necessary to blend it with another polymer or add fillers to improve its properties. Polypropylene (PP) could be a suitable polymer to blend with TPS once it exhibits an attractive combination of low weight and density, low cost and heat distortion temperature (Manias et al., 2001).

Although nanocomposites of TPS and PP-g-MA have been developed, there is a lack on the effect of clay mineral addition on the enhancement of phase compatibility, improvement of TPS/PP mechanical properties as well as nanocomposites biodegradability. Therefore, different ratios of TPS/PP-g-MA (1:2; $1: 1$ and $2: 1)$ without and with 5 wt.\% of Cloisite 30B were prepared by melt compounding using an internal mixer. The morphology and clay mineral dispersion of the nanocomposites were observed by scanning electron microscopy (SEM), scanning transmission electron microscopy (STEM) and X-ray diffraction (XRD). While dynamic mechanical analysis (DMA) was used to investigate the mechanical performance, composting tests allowed assessing biodegradability. 
Table 1

Sample codes and compositions.

\begin{tabular}{|c|c|c|c|}
\hline Sample code & TPS (wt.\%) & PP-g-MA (wt.\%) & C30B (wt.\%) \\
\hline TPS & 100 & & \\
\hline TPS/C30B & 95 & & 5 \\
\hline TPS/PP-g-MA (2:1) & 66.7 & 33.3 & \\
\hline TPS/PP-g-MA (2:1)/C30B & 63.3 & 31.7 & 5 \\
\hline TPS/PP-g-MA (1:1) & 50 & 50 & \\
\hline TPS/PP-g-MA (1:1)/C30B & 47.5 & 47.5 & 5 \\
\hline TPS/PP-g-MA (1:2) & 33.3 & 66.7 & \\
\hline TPS/PP-g-MA (1:2)/C30B & 31.7 & 63.3 & 5 \\
\hline PP-g-MA & 100 & & \\
\hline PP-g-MA/С $30 \mathrm{~B}$ & 95 & & 5 \\
\hline
\end{tabular}

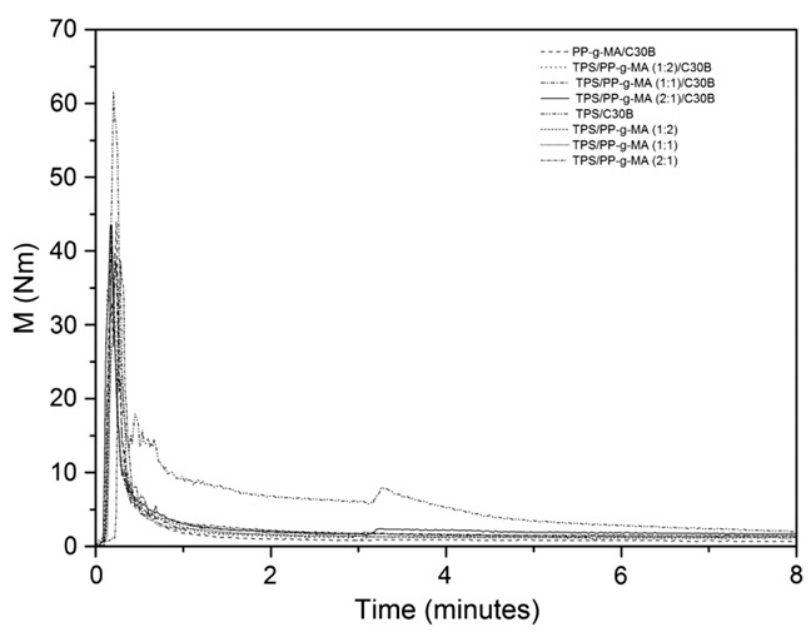

Fig. 1. Torque measurements obtained during materials preparation in a batch mixer.

\section{Experimental}

\subsection{Materials}

Thermoplastic starch (TPS), Mater-Bi®, was supplied by Novamont. Mater-Bi ${ }^{\circledR}$ is commercially available as blends of corn starch/PCL 30/70 (wt.\%). Polypropylene-grafted-maleic anhydride (PP-g-MA, Poly-bond 3200 ) with a melting temperature around $160{ }^{\circ} \mathrm{C}$ and a MA content of 1 wt.\%, was supplied by Crompton. Cloisite ${ }^{\circledR}$ 30B (C30B) a montmorillonite (MT) modified with a quaternary ammonium salt (MT2EtOH = methyl tallow bis-2-hydroxyethyl ammonium chloride) was used as received.

\subsection{Preparation of bio-based polymer nanocomposites}

Compounds with different ratios of TPS and PP-g-MA (1:2; $1: 1$ and $2: 1$ ) containing $\mathrm{C} 30 \mathrm{~B}$ were prepared in the melt, in a Haake batch mixer (Rheocord 90; volume $60 \mathrm{~cm}^{3}$ ), equipped with two rotors running in a counter-rotating way. The rotor speed was $50 \mathrm{rpm}$ and the set temperature was $180^{\circ} \mathrm{C}$. The following procedure was adopted to prepare the nanocomposites, a homogeneous pre-mixture of TPS and PP-g-MA pellets were introduced into the hot mixer. After melting, 5 wt.\% of C30B, dried at $105{ }^{\circ} \mathrm{C}$ under vacuum for at least $24 \mathrm{~h}$, was added. The total sample was removed after 10 min of mixing. Table 1 presents the composition of the prepared samples.

\subsection{Characterization}

\subsection{1. $X R D$}

X-ray diffraction measurements (XRD) were performed to investigate the intercalation and exfoliation of C30B. XRD spectra of the samples were obtained at room temperature using a diffractometer (AXS Nanostar-D8 Discover, Bruker) equipped with a CuK $\alpha$ generator $(\lambda=$ $1.5406 \AA$ ) at $40 \mathrm{~mA}$ and $40 \mathrm{kV}$. Data were collected in a range of 0.08 to $10^{\circ}$ with a step size of $0.01^{\circ}$ and a counting time of $2 \mathrm{~s}$ per step. The C30B powder or disks were analyzed directly, whereas the nanocomposite samples were previously compression molded in a press (30 ton for $1 \mathrm{~min}$ at $180^{\circ} \mathrm{C}$ ) into disks with $20 \mathrm{~mm}$ diameter and a thickness of $4 \mathrm{~mm}$. Bragg's law ( $\mathrm{n}=2 d_{001} \sin \theta$ ) was used to determine the clay mineral interlayer distance $d_{001}$, where $d$ is the spacing between silica layers of the C30B (also called interlayer spacing), $\lambda$ the wavelength of X-ray on the silica layer, and $\mathrm{n}$ is a whole number which represents the order of diffraction, taken 1 in our calculations.

\subsubsection{Microscopy}

Scanning electron microscopy (SEM) was carried out to analyze the nanocomposites morphology. SEM analysis was performed in a Leica Cambridge S360 microscope. The samples were previously fractured in liquid nitrogen and coated with a gold thin film.

Scanning transmission electron microscopy (STEM) observations of the nanocomposites were performed with a NanoSEM Nova200 (FEI), using an acceleration voltage of $15 \mathrm{kV}$. Ultra-thin sections of approximately $80 \mathrm{~nm}$ were cut from samples collected from the Haake batch mixer at $-20^{\circ} \mathrm{C}$, using a UC6 ultramicrotome (Leica) equipped with a diamond knife.

\subsubsection{DMA}

Dynamic mechanical analysis (DMA) experiments were performed in a TRITON apparatus in tension mode under the following parameters: frequency $=1 \mathrm{~Hz}$; temperature scan rate $=2{ }^{\circ} \mathrm{C} / \mathrm{min}$; temperature range $=-70$ to $100{ }^{\circ} \mathrm{C}$; free length $=15 \mathrm{~mm}$ and value tension $=$ $15 \mu \mathrm{m}$. The equipment was calibrated according to the standard procedure. The tests were performed on $3 \mathrm{~mm} \times 30 \mathrm{~mm}$ rectangular samples in a longitudinal direction. At least two specimens of each sample were tested. Prior to mechanical measurements, plates were prepared by compression molding in a press ( $30 \mathrm{t}$ for $1 \mathrm{~min}$ at $180^{\circ} \mathrm{C}$ ).

\subsubsection{Biodegradability assessment in compost}

Biodegradation in compost was performed on TPS, TPS/C30B, TPS/ PP-g-MA (1:1) and TPS/PP-g-MA (1:1)/C30B samples at $40{ }^{\circ} \mathrm{C}$. Using

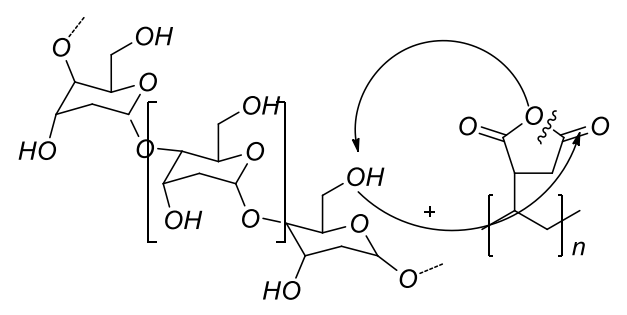

TPS

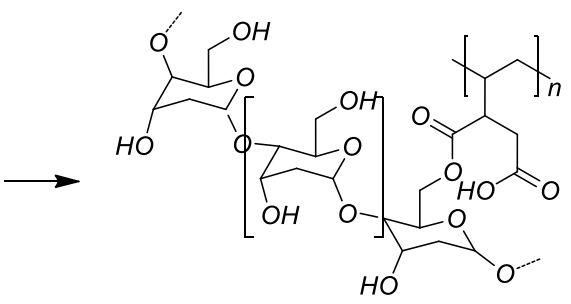

TPS-g-PP

Scheme 1. Possible reaction mechanism of grafting of TPS onto PP-g-MA. 


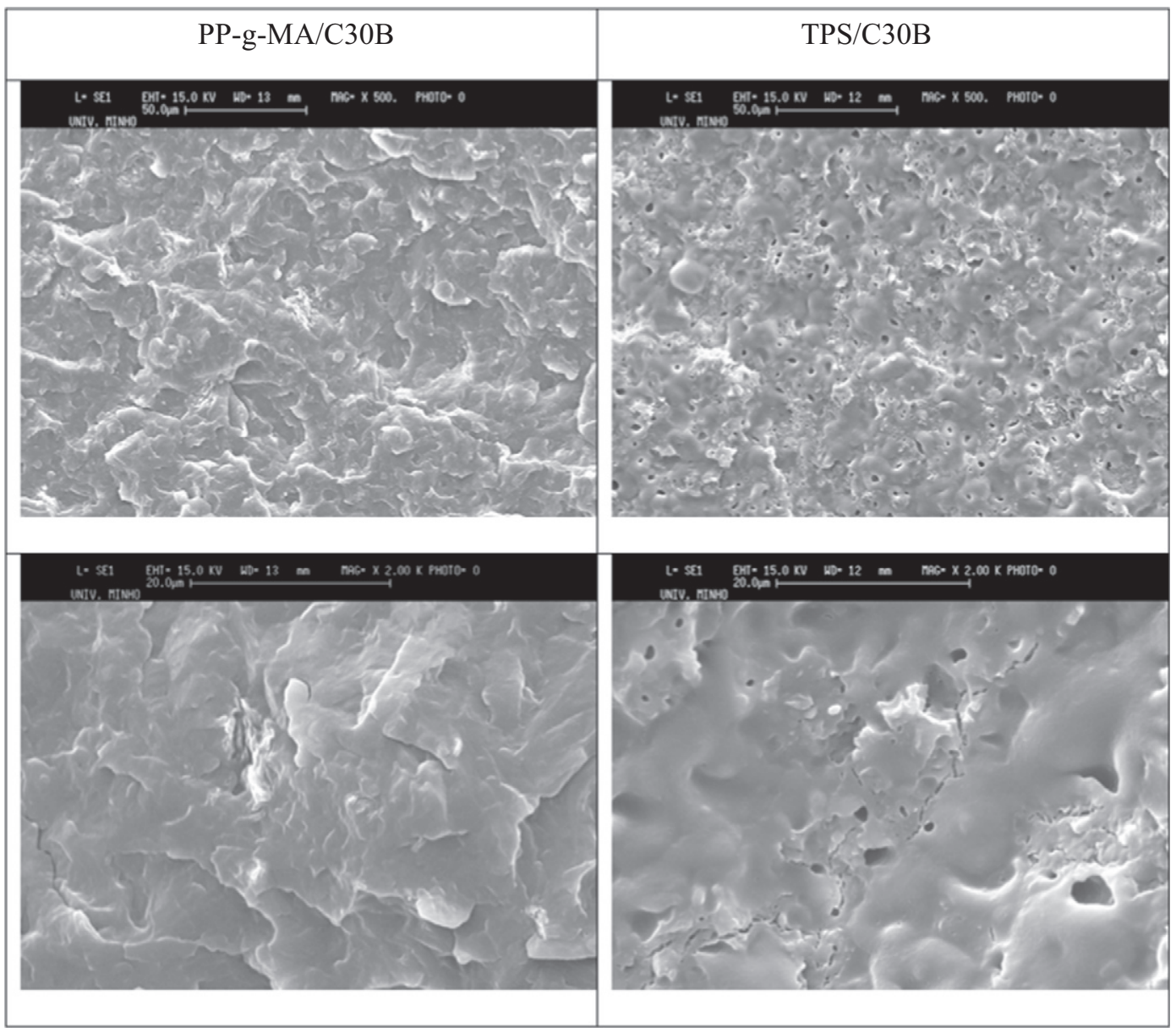

Fig. 2. Morphology of PP-g-MA/C30B and TPS/C30B nanocomposites (5 wt.\% of C30B).

rectangular samples $(25 \times 25 \times 0.125 \mathrm{~mm})$, they were placed in a composting medium made of soil, activated sludges from waste water treatment, straw and animal manure. The composting medium was kept in a relative humidity of approximately $50-70 \%$. Around 15 samples of each material were horizontally buried at $6-8 \mathrm{~cm}$ depth to guarantee aerobic degradation conditions at a horizontal distance of 5-6 cm between samples according to Fukushima et al. (2009). At selected times (every 3 weeks), samples were collected, cleaned and dried at room temperature until constant weight was reached. Based on the sample weight before and after composting, the average percentage of residual weight for each material was calculated.

\section{Results and discussion}

The torque measurements recorded during the preparation of the different samples are shown in Fig. 1. The torque increases in the first few seconds after the addition of the polymers and then decreased as the polymers started to melt. After 3 min of mixing, the C30B was added and for TPS/PP-g-MA (1:1) and TPS an increase in torque value can be observed. This change can be especially noticed for TPS, when compared to the torque of pristine polymer. The increase in torque after the clay mineral addition, gives evidence that is dispersed in the polymeric matrices.

The organically modified clay mineral used in this work, C30B, has two hydroxyl groups $(-\mathrm{OH})$, which can react with the anhydride group of PP-g-MA (-CO-O-CO-) to give an ester (-COO-) and an acid $(-\mathrm{COOH})$. The acid group can then react with the second hydroxyl group (or coming from another part of the C30B structure) to form a secondary ester and a water molecule (Tessier et al., 2012). However, the hydroxyl groups presented in starch molecules, which are mainly constituted of amylose and amylopectin, can also react with the carbon$\mathrm{yl}$ of the maleic anhydride of the PP-g-MA to form TPS-g-PP copolymer (Scheme 1).

When TPS and PP-g-MA in different ratios are melt mixing with C30B in the mixer, both reactions aforementioned can occur. These chemical reactions allow the formation of TPS-g-PP copolymer promoting an interpenetrating network creation. Such effect could improve clay mineral layers separation enhancing intercalation and/or exfoliation.

The first level of morphological analysis consisted in evaluating the dispersion of C30B in PP-g-MA and TPS and the effect of clay mineral addition on TPS/PP-g-MA (1:2), TPS/PP-g-MA (1:1) and TPS/PP-g-MA (2:1) blend morphologies. Fig. 2 presents SEM micrographs of PP-g$\mathrm{MA} / \mathrm{C} 30 \mathrm{~B}$ and TPS/C30B nanocomposites. Both samples, at the two magnifications, do not present any visible clay layers, suggesting a homogeneous dispersion of the $\mathrm{C} 30 \mathrm{~B}$ in the polymer. This can be due to the interaction between the hydroxyl group of the organoclay and functional groups of the polymers.

Blends morphologies with different ratios of TPS/PP-g-MA without and with $\mathrm{C} 30 \mathrm{~B}$ are presented in Figs. 3 to 5 . The clay mineral addition to TPS/PP-g-MA (1:2) (Fig. 3), TPS/PP-g-MA (1:1) (Fig. 4) and TPS/PPg-MA (2:1) (Fig. 5) blends, has a strong influence on their morphology. SEM micrographs of the nanocomposites exhibit a more homogeneous morphology and lower size of the dispersed phase when compared to the blends morphology (Figs. 3-5). SEM results evidence that the polar character of organoclay C30B is a key factor for the higher interaction with the polymer matrix. Compatibilization can also be enhanced if the reaction proposed in Scheme 1 occurs. Therefore both clay mineral and copolymer can act as a compatibilizer, decreasing the interfacial 


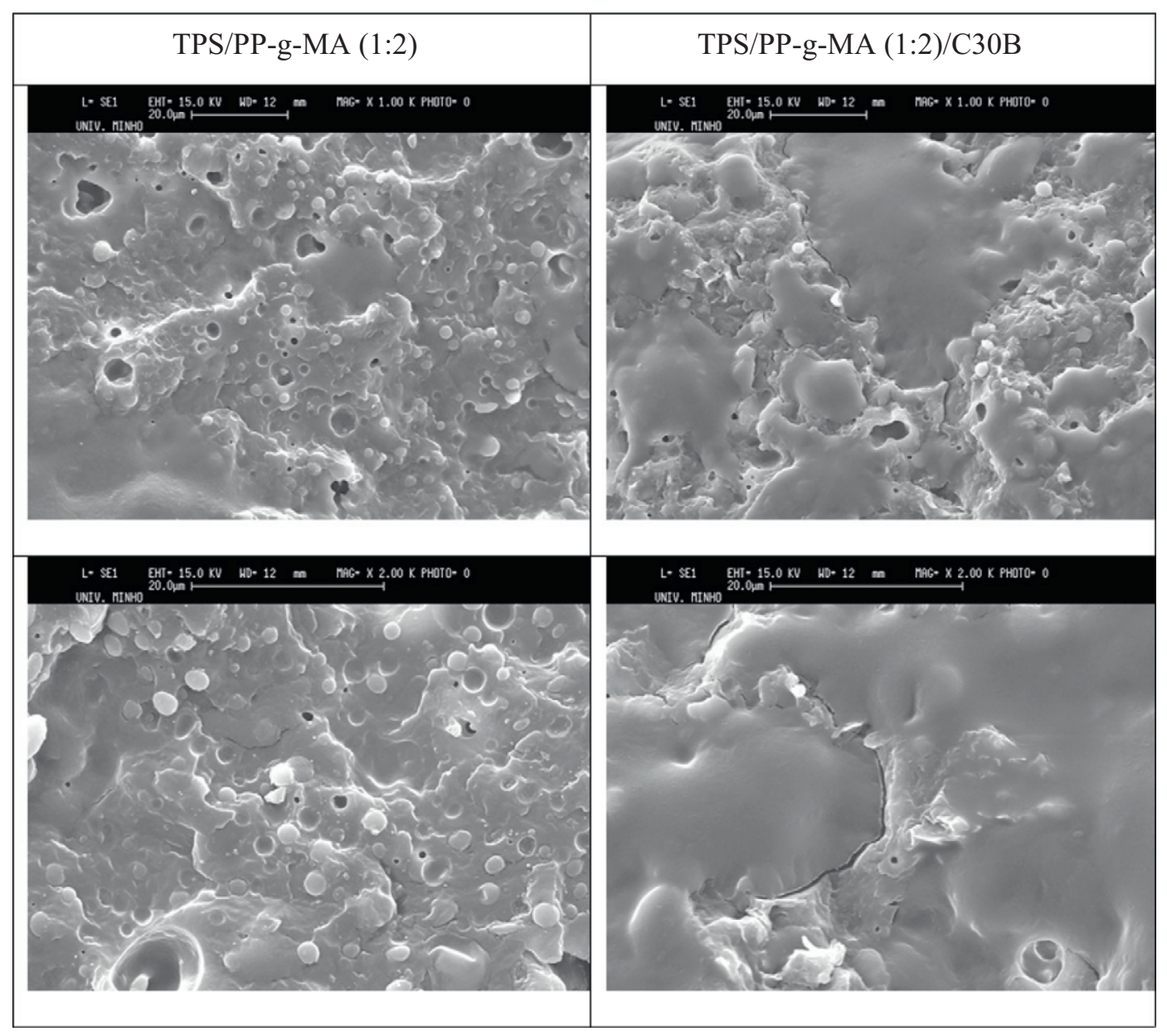

Fig. 3. Morphology of TPS/PP-g-MA (1:2) blend and TPS/PP-g-MA (1:2) with 5 wt.\% of C30B.

tension and the particle size. C30B agglomerates cannot be detected, which points out a good dispersion level.

Fig. 6 presents the X-ray diffraction (XRD) analysis of neat C30B, PPg-MA/C30B, TPS/PP-g-MA (1:2)/C30B, TPS/PP-g-MA (1:1)/C30B, TPS/ PP-g-MA (2:1)/C30B and TPS/C30B. A significant peak shift to lower angles, relatively to $\mathrm{C} 30 \mathrm{~B}$, can be noticed for all samples indicating an intercalated and/or exfoliated (at least partially) dispersion of the clay layers (Fig. 6). Moreover, peak shape also changed, being the height very small and the width broad in some cases, indicating a very good dispersion of the clay layers. Based on the XRD results, the interlayer space was determined using Bragg's law for the organoclay and the nanocomposites. The mean interlayer spacing of the (001) plane $\left(\mathrm{d}_{001}\right)$ for the neat C30B solid obtained was $1.86 \mathrm{~nm}\left(2 \theta=4.75^{\circ}\right)$ (Table 2). For the different matrices (PP-g-MA, blends of TPS/PP-g-MA and TPS) weaker peaks are observe between $3.57 \leq 2 \theta \geq 3.90$ ( $2 \theta$ for neat $\mathrm{C} 30 \mathrm{~B}$ located around $\left.4.75^{\circ}\right)$. The X-ray diffraction results (Table 2) indicate an increase of the interlayer spacing due to polymer intercalation within clay layers, in agreement with the SEM data, Figs. 2-5. Among all nanocomposites, TPS/PP-g-MA (1:1) demonstrated to be the one with higher clay interlayer distance $\left(d_{001}=2.47 \mathrm{~nm}\right)$.

To further confirm the nanostructure of the prepared nanocomposites STEM analysis were carried out. Fig. 7 depicts the STEM micrographs of PP-g-MA and TPS nanocomposites. It is possible to verify that the clay layers (dark traces) are homogeneously dispersed in the TPS matrix, due to the higher affinity between polar functional groups of $\mathrm{C} 30 \mathrm{~B}$ and the thermoplastic starch phase. Moreover, two phases can be observed in STEM image of TPS/C30B, which is due to the fact that this commercial TPS is a blend of corn starch and PCL. PP-g-MA/C30B nanocomposite, STEM micrograph exhibits intercalated structures but not so homogeneously dispersed as in the TPS matrix (Fig. 7). These results are in agreement with the XRD findings.

Blends morphologies, for TPS/PP-g-MA (1:1) and for TPS/PP-g-MA (1:1)/C30B, were also analyzed by STEM (Fig. 8). Two polymeric phases are clearly detected in both systems. However, the dispersed phase is smaller in the TPS/PP-g-MA nanocomposites and C30B dispersion occurs mainly in the matrix and at the interface. Other authors have already observed the affinity of C30B to TPS, which is explained by the interaction between the hydrophilic character of TPS and the polar character of the modified C30B (Tessier et al., 2012). Based on the decrease of the particle size, although the TPS-g-PP copolymer formed at the interface (Scheme 1) can decrease the size of the dispersed phase, the effect of the clay mineral as compatibilizer had a major effect. Clearly, the $\mathrm{C} 30 \mathrm{~B}$ addition improved the interfacial adhesion and contributed for the decrease of the dispersed phase.

Since the morphology has a strong effect on mechanical properties, Fig. 9 (a to c) shows the DMA results of TPS, PP-g-MA, TPS/PP-g-MA (1:1) and the corresponding nanocomposites. Each one presents the plot of temperature dependence of storage modulus $\left(G^{\prime}\right)$, loss modulus $\left(G^{\prime \prime}\right)$ and tangent $\delta(\tan \delta)$. As expected the addition of clay mineral increases the storage modulus of all systems. TPS/C30B nanocomposite exhibits the higher increase of dynamic storage modulus (28\% more than pristine TPS) (Fig. 9a). For the remaining nanocomposites the increase of dynamic storage modulus was lower (about 11\% in each case). The effect of the $\mathrm{C} 30 \mathrm{~B}$ addition to polymer matrices results in an increase of the modulus or stiffness via reinforcement mechanisms described by theories for composites (Fornes and Paul, 2003; Lee and Paul, 2005). A possible explanation for the improvement of the storage modulus even at temperatures above the room temperature could be the 


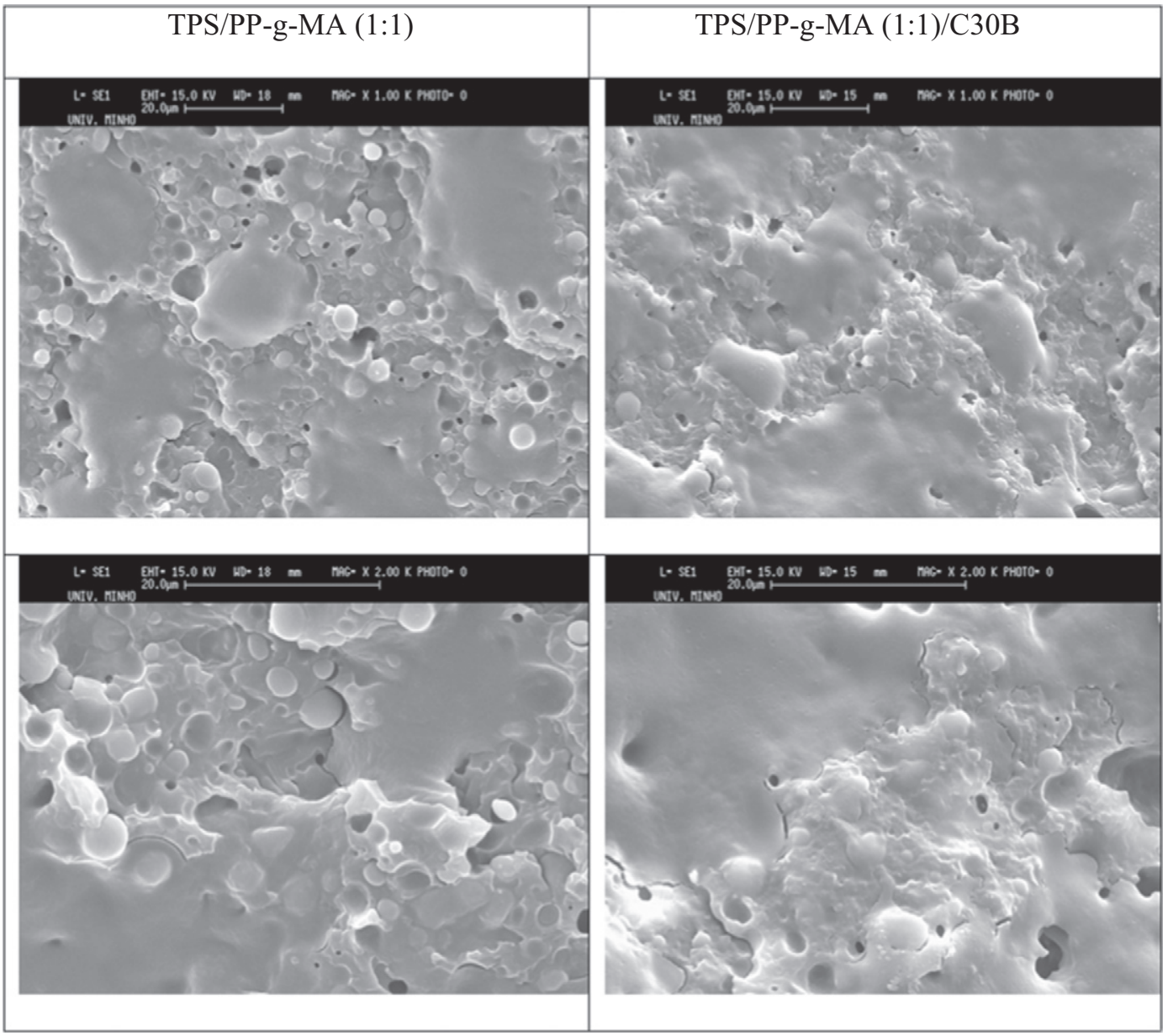

Fig. 4. Morphology of TPS/PP-g-MA (1:1) blend and TPS/PP-g-MA (1:1) with 5 wt.\% of C30B.

formation of a three-dimensional network of interconnected long silicate layers, strengthening the material through mechanical percolation (Lan et al., 1994). Moreover, the addition of PP-g-MA to TPS increases the TPS modulus at higher temperatures.

While $\tan \delta$ of neat PP-g-MA presents one transition peak at $5{ }^{\circ} \mathrm{C}\left(\mathrm{T}_{g}\right)$ (Fig. 9b), the nanocomposite shows a shift to $9^{\circ} \mathrm{C}$ and a new transition peak at $45^{\circ} \mathrm{C}$. This can be due to the effect of the clay mineral in the crystallinity of PP-g-MA. For TPS and respective nanocomposite, two transitions peaks $\left(0\right.$ and $\left.70^{\circ} \mathrm{C}\right)$ can be detected, being $T_{g}$ slightly lower $\left(62^{\circ} \mathrm{C}\right)$ in TPS nanocomposite than for pristine TPS. TPS containing native potato starch/water/glycerol (5/2/3 by wt. ratio) revealed two thermal relaxations, a low temperature transition between $-60{ }^{\circ} \mathrm{C}$ and $-50{ }^{\circ} \mathrm{C}$, attributed to the glycerol-rich (Park et al., 2003). Since our TPS material has a different composition the glycerol-rich phase was not detected, only the starch-rich phase.

The $\tan \delta$ behavior of the nanocomposite in Fig. 9c shows that the three transition temperatures $\left(5,65,90^{\circ} \mathrm{C}\right)$ of the neat TPS/PP-g-MA (1:1) are shifted to lower temperatures due to repulsion forces between the matrix and the polar organoclay. TPS/PP-g-MA (1:1) nanocomposite presents a new temperature transition peak at $43^{\circ} \mathrm{C}$, which correspond to the peak detected for PP-g-MA at $45^{\circ} \mathrm{C}$. This small shifted to lower temperatures can be related to changes in molecular organization.

Biodegradation in compost for TPS, TPS/C30B, TPS/PP-g-MA (1:1) and TPS/PP-g-MA (1:1)/C30B samples was performed during 14 weeks at 40 ${ }^{\circ} \mathrm{C}$. Samples were collected, cleaned, dried and weighted after 3, 9 and 14 weeks (Fig. 10).

After 98 days of incubation in composting the results revealed that TPS and TPS/C30B samples have the higher biodegradability when compared with TPS/PP-g-MA (1:1) and TPS/PP-g-MA (1:1)/C30B. TPS/C30B showed the highest weight loss, which can be associated with higher biodegradation rate. The addition of the $\mathrm{C} 30 \mathrm{~B}$ also had a positive effect of the TPS/PP-g-MA blend biodegradability, the nanocomposites exhibited higher weight variation along the time. Generally the presence of clay minerals allows the acceleration of the biodegradation process, but it also depends on the bio-based polymer compositions. The TPS material, consumed by microbial action, accelerates the disintegration or fragmentation of polymer chain by producing pores in the materials, which makes it weak and increases its biodegradability. This process is quite slow, it can accelerate if the ratio starch/other materials, as additives, exceeds 60 wt.\% in TPS polymer (Siracusa et al., 2008).

\section{Conclusion}

The effect of C30B addition to TPS/PP-g-MA blends on morphology, mechanical properties and biodegradability was investigated. The characterization techniques used demonstrated that the clay mineral was well dispersed in the polymeric matrix and contributed to an increase in polymer compatibility, mechanical properties and biodegradability.

Therefore clay minerals, which can be obtained from natural resources, can be efficiently used to improve the properties of bio-based materials and contribute to sustainability.

\section{Acknowledgment}

The authors acknowledge the n-STeP - Nanostructured systems for Tail, with reference NORTE-07-0124-FEDER-000039, supported by the Programa Operacional Regional do Norte (ON.2), PEst-C/CTM/LA0025/ 2013 (Strategic Project - LA 25 - 2013-2014). 


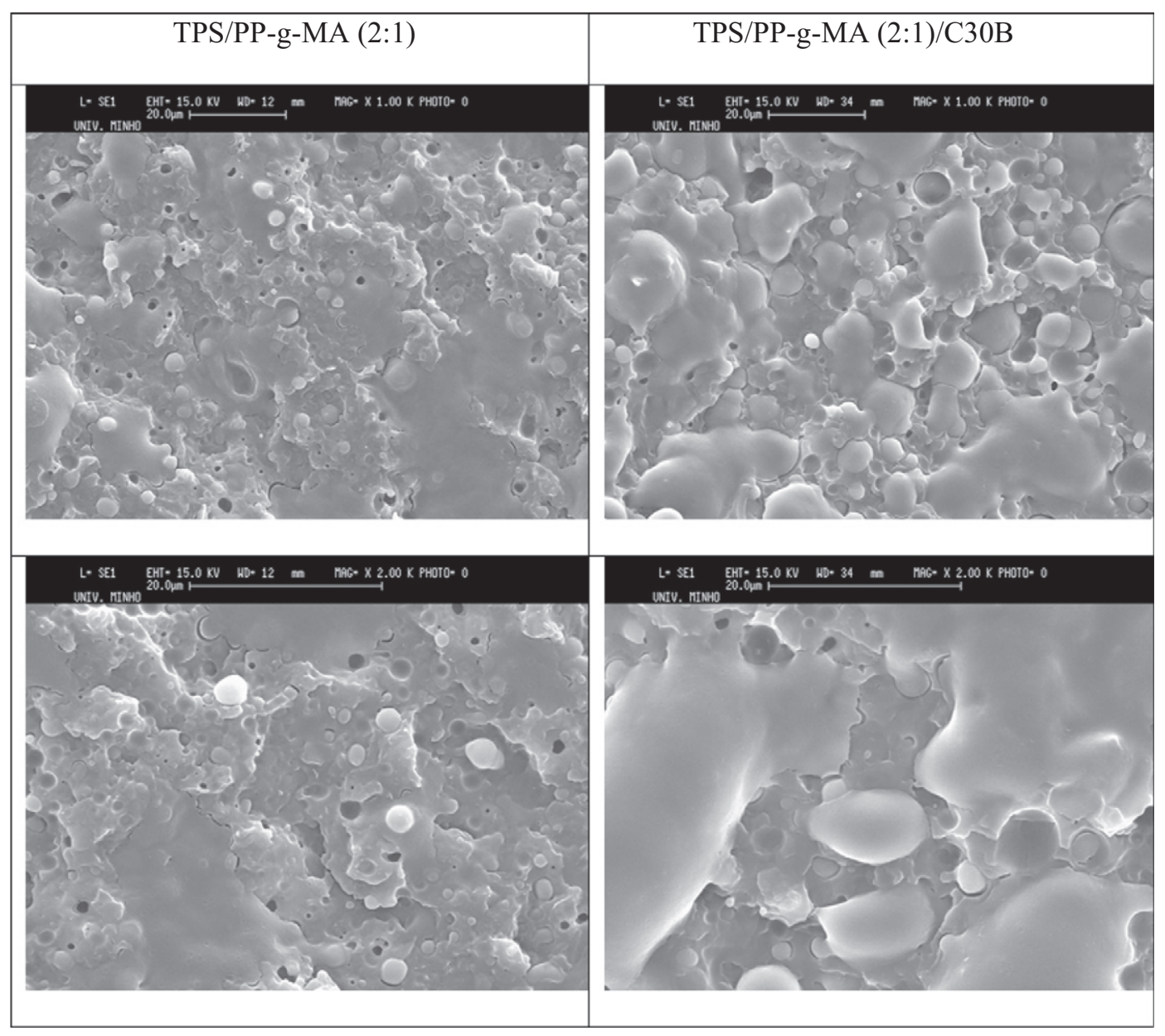

Fig. 5. Morphology of TPS/PP-g-MA (2:1) blend and TPS/PP-g-MA (2:1) with 5 wt.\% of C30B nanocomposites.

\section{References}

Fornes, T.D., Paul, D.R., 2003. Modeling properties of nylon 6/clay nanocomposites using composite theories. Polymer 44 (17), 4993-5013.

Fukushima, K., Abbate, C., Tabuani, D., Gennari, M., Camino, G., 2009. Biodegradation of poly(lactic acid) and its nanocomposites. Polym. Degrad. Stab. 94 (10), 1646-1655.

Hottle, T.A., Bilec, M.M., Landis, A.E., 2013. Sustainability assessments of bio-based polymers. Polym. Degrad. Stab. 98 (9), 1898-1907.

Kráčalík, M., Studenovský, M., Mikešová, J., Kovářová, J., Sikora, A., Thomann, R., Friedrich, C., 2007. Recycled PET-organoclay nanocomposites with enhanced processing properties and thermal stability. J. Appl. Polym. Sci. 106 (3), 2092-2100.

Lan, T., Kaviratna, P.D., Pinnavaia, T.J., 1994. On the nature of polyimide-clay hybrid composites. Chem. Mater. 6 (5), 573-575.

Lee, K.Y., Paul, D.R., 2005. A model for composites containing three-dimensional ellipsoidal inclusions. Polymer 46 (21), 9064-9080.

Manias, E., Touny, A., Wu, L., Strawhecker, K., Lu, B., Chung, T.C., 2001. Polypropylene/ montmorillonite nanocomposites. Review of the synthetic routes and materials properties. Chem. Mater. 13 (10), 3516-3523.
Mohammadi Nafchi, A., Moradpour, M., Saeidi, M., Alias, A.K., 2013. Thermoplastic starches: properties, challenges, and prospects. Starch - Stärke 65 (1-2), 61-72.

Ojijo, V., Sinha Ray, S., 2013. Processing strategies in bionanocomposites. Prog. Polym. Sci. 38 (10-11), 1543-1589.

Park, H.-M., Lee, W.-K., Park, C.-Y., Cho, W.-J., Ha, C.-S., 2003. Environmentally friendly polymer hybrids. Part I Mechanical, thermal, and barrier properties of thermoplastic starch/clay nanocomposites. J. Mater. Sci. 38 (5), 909-915.

Rhim, J.-W., Park, H.-M., Ha, C.-S., 2013. Bio-nanocomposites for food packaging applications. Prog. Polym. Sci. 38 (10-11), 1629-1652.

Siracusa, V., Rocculi, P., Romani, S., Rosa, M.D., 2008. Biodegradable polymers for food packaging: a review. Trends Food Sci. Technol. 19 (12), 634-643.

Tang, X., Alavi, S., 2011. Recent advances in starch, polyvinyl alcohol based polymer blends, nanocomposites and their biodegradability. Carbohydr. Polym. 85 (1) 7-16.

Tessier, R., Lafranche, E., Krawczak, P., 2012. Development of novel melt-compounded starch-grafted polypropylene/polypropylene-grafted maleic anhydride/organoclay ternary hybrids. Exp. Polym. Lett. 6 (11), 937-952. 


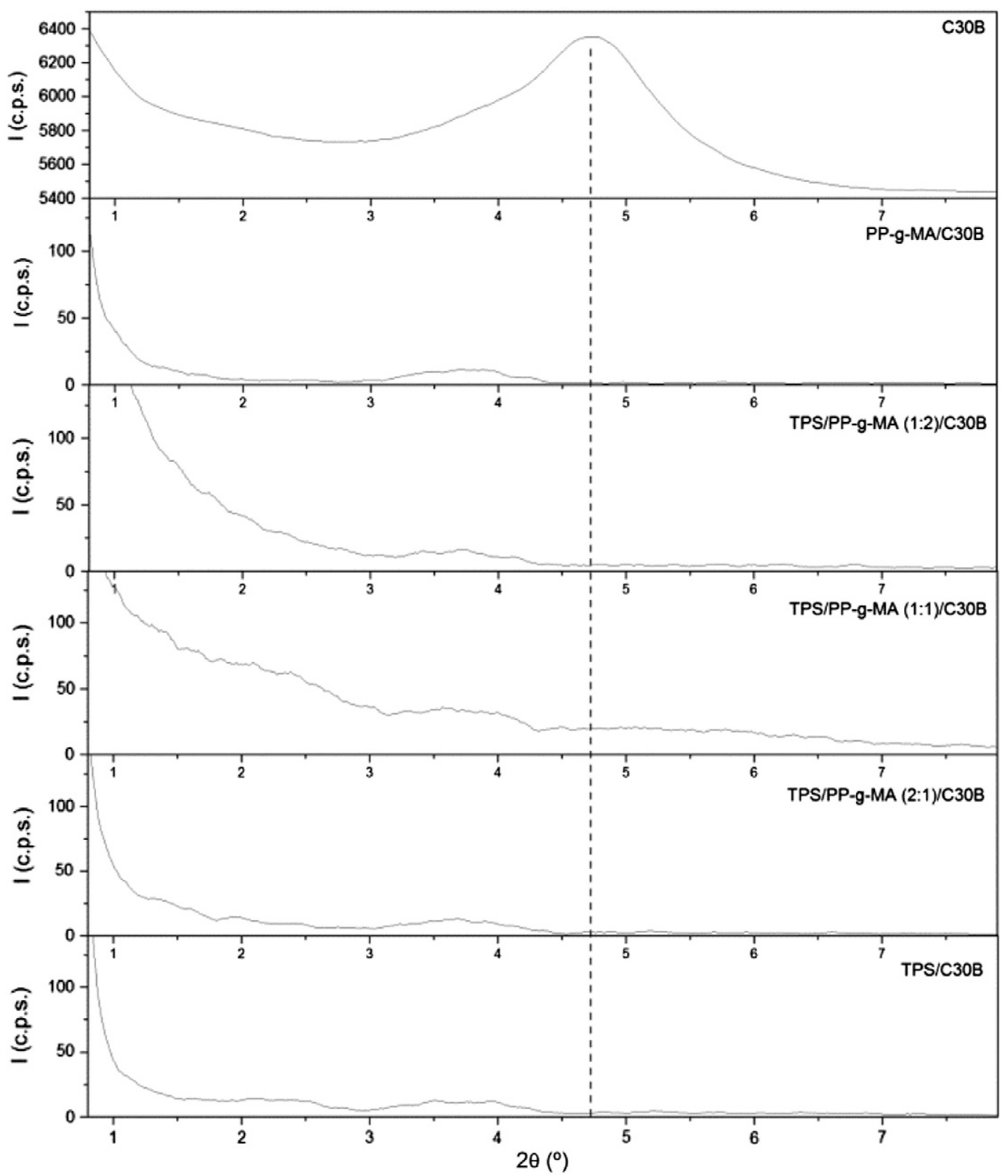

Fig. 6. X-ray diffraction patterns of C30B and nanocomposites.

Table 2

Clay interlayer distance $\left(d_{001}\right)$ of C30B and nanocomposites (using Bragg's law ( $\mathrm{n}=2 d_{001}$ $\sin \theta)$.

\begin{tabular}{lll}
\hline & $2 \theta\left({ }^{\circ}\right)$ & $d_{001}(\mathrm{~nm})$ \\
\hline PP-g-MA/C30B & 3.90 & 2.27 \\
TPS/PP-g-MA (1:2)/C30B & 3.72 & 2.37 \\
TPS/PP-g-MA (1:1)/C30B & 3.57 & 2.47 \\
TPS/PP-g-MA (2:1)/C30B & 3.69 & 2.39 \\
TPS/C30B & 3.64 & 2.43 \\
C30B & 4.75 & 1.86 \\
\hline
\end{tabular}




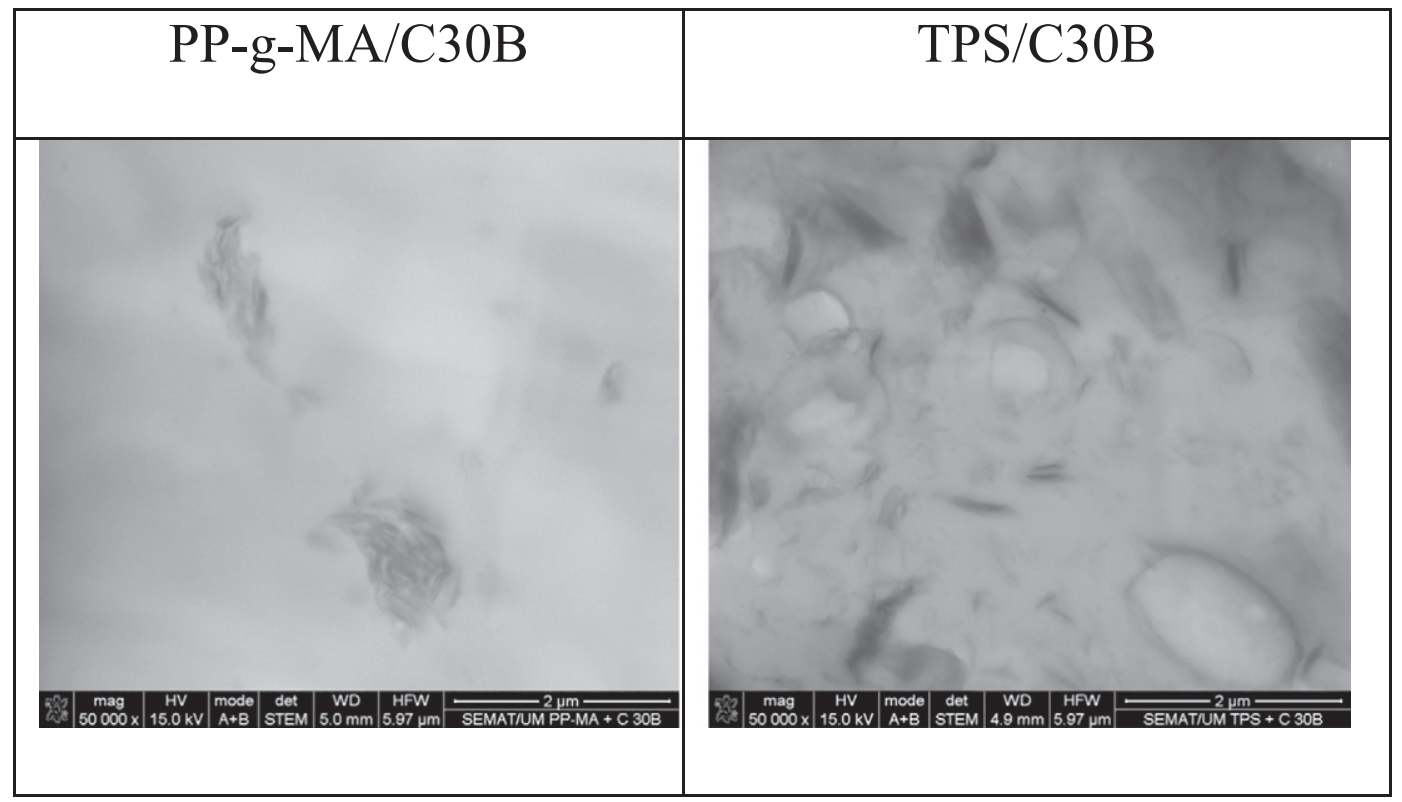

Fig. 7. STEM micrographs of PP-g-MA and TPS nanocomposites.

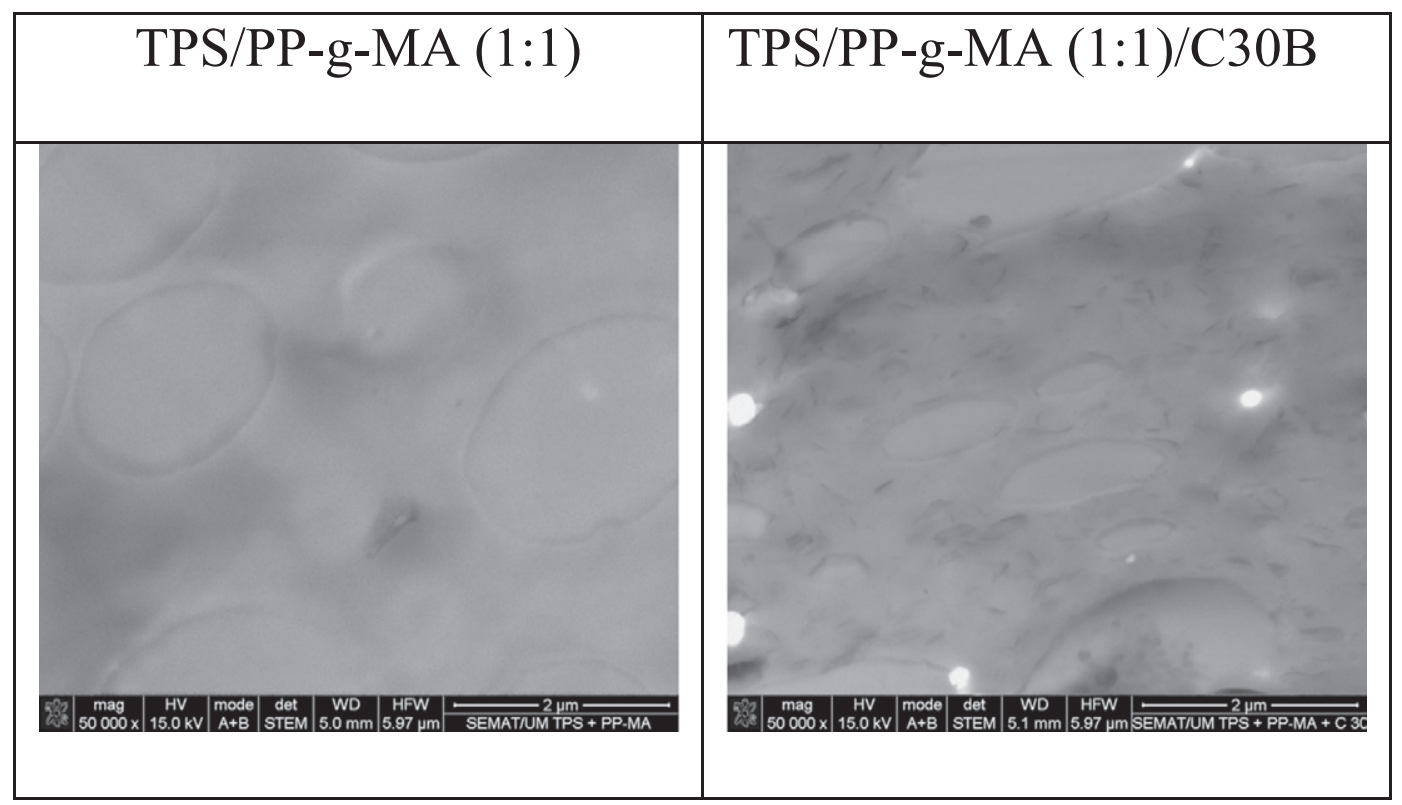

Fig. 8. STEM micrographs of neat TPS/PP-g-MA (1:1) and TPS/PP-g-MA (1:1)/C30B.
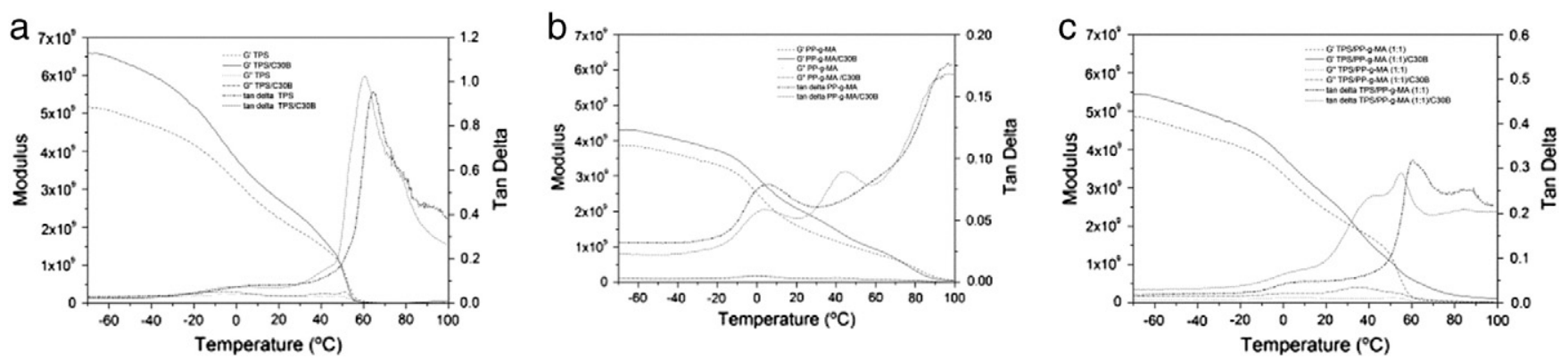

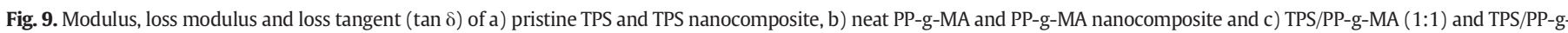
MA (1:1) nanocomposite. 


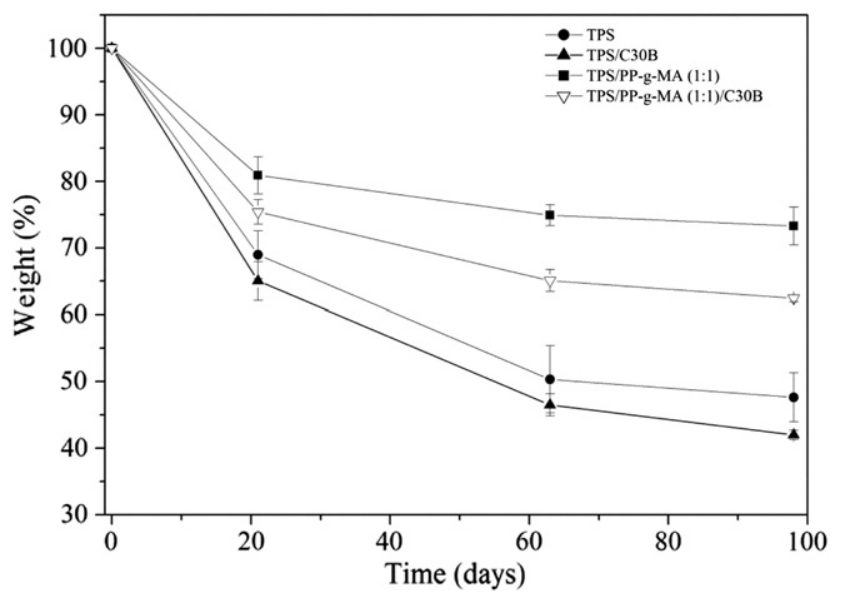

Fig. 10. Weight loss of TPS, TPS/C30B, TPS/PP-g-MA (1:1) and TPS/PP-g-MA (1:1)/C30B during 14 weeks of degradation in compost at $40^{\circ} \mathrm{C}$. 